\title{
Energy levels and structural properties of compressed hydrogen atom under Debye screening
}

\author{
B. Saha ${ }^{1}$, P. K. Mukherjee ${ }^{1}$, and G. H. F. Diercksen ${ }^{2}$ \\ 1 Department of Spectroscopy, Indian Association for the Cultivation of Science, Jadavpur, Kolkata-700 032, India \\ 2 Max-Planck-Institut für Astrophysik, Karl-Schwarzschild Strasse 1, 85741 Garching, Germany
}

Received 10 June 2002 / Accepted 16 September 2002

\begin{abstract}
Time dependent variation perturbation calculations have been performed for estimating the transition energies, oscillator strengths and transition probability values for a few dipole allowed states of compressed hydrogen atom confined in a weakly coupled plasma. The compression is obtained by embedding the atom at the centre of an impenetrable spherical box. The dipole polarizability of the atom is evaluated at each confinement radius with respect to different plasma screening parameters. The effect of pressure due to spatial confinement on the dipole polarizability and other atomic properties is analyzed. Results obtained are useful for the diagnostic determination of astrophysical and laboratory plasmas and for the calculation of collision rate coefficients needed for computing opacity of stellar envelopes - a quantity of importance in the context of stellar structure and pulsations.
\end{abstract}

Key words. atomic data - atomic processes

\section{Introduction}

In recent years the analysis of the spectral properties of confined atomic systems such as those of atoms embedded in liquid helium has gained considerable momentum (Tabbert 1997) firstly, because of the availability of current implantation technique like ion beam method (Gordon 1974, 1983, 1993), laser sputtering and ablation technique (Bauer 1990; Yabuzaki 1992; Arndt 1993; Tabbert 1994; Beijersbergen 1993; Kinoshita 1995) and secondly due to accurate methods available for estimating the spectral line shifts (Günther 1995; Tabbert 1995; Kanorski 1994). Although a host of experimental data for different atoms are currently available, very few methods exist for theoretical estimation of the spectral line shifts and other properties under such confinement. In the so called standard bubble method (SBM), the total Hamiltonian is expressed as the sum of pair interaction between helium atoms, between impurity atom and each of the helium atoms and the Hamiltonian of the free atom itself. The total impurity helium interaction is derived from individual impurity-helium interaction term. First order perturbation theory is being utilized to calculate the energy shift. The method has been applied in a number of cases (Hiroike 1965; Hikman 1975; Bauer 1990; Beau 1996). Basically the problem is similar to that of studying the spectra of compressed atoms like that trapped in zeolite, fullerenes or under high pressure (Jaskolski 1996; Connerade 2000; Gupta 1982). As ab-initio calculations for atoms in such an environment is very complicated, the problem of the impurity atom

Send offprint requests to: P. K. Mukherjee, e-mail: sppkm@mahendra.iacs.res.in embedded in liquid helium may alternatively be looked upon using a suitable model in the following manner. Experimental observations indicate that (i) electrons in superfluid helium are highly mobile and (ii) because of the inert nature of helium the atomic charge density has extremely low penetration probability beyond the surrounding liquid helium cage due to strong Pauli repulsion. Hence one can tentatively assume the foreign atom to be embedded in a charge neutral environment which mimics a plasma with a finite cage boundary. With this viewpoint we propose a model of studying atoms inside such an environment which, on one hand assumes screening of the potential due to plasma, and secondly uses an altered boundary condition which suits the physical description. We consider at present the surrounding plasma to be sufficiently weak so as to produce screened coulomb interaction between point charges. The effect of this so called Debye plasma on atomic energy levels and other properties has been analyzed earlier in a limited manner (Winkler 1996; Ray 1998a, 1998b). The overall effect of this screened Coulomb potential is to reduce the binding energy and to push the system towards gradual instability with increase of screening. The screening constant is a function of temperature and number density of the plasma and different plasma conditions can be simulated by changing suitably the screening parameters. Study of laser produced and Tokamak plasmas requires an understanding of the electronic spectrum of an ion as a function of electron density and temperature of the plasma. The screening is important in interpreting the disappearance of spectral lines near the series limit in astrophysical observations, in laboratory and astrophysical plasma diagnostics, in calculating partition functions in 
thermodynamics, in the calculation of collision rate coefficients, all of which require the shifted energy levels and connected properties. Such calculations have also applications in astrophysical phenomena like the mass radius relation in the theory of white dwarfs and in the determination of the rate of escape of stars from galactic and globular clusters, understanding the interior of giant planets (Varshni 1997, 1998) and in estimating stellar opacities (Seaton 1987). The study of the properties of a confined atom in an impenetrable spherical box has, however, a long history. Such studies originated with a model due to Michels et al. (1937) who studied the effect of pressure by enclosing an atom in a spherical box. Sommerfeld \& Welker (1938) carried out investigations on the energy levels of hydrogen in a spherical box. Subsequently, a number of calculations (Ley-Koo 1979; Ludena 1977, 1978; Marin \& Cruz 1991a, 1991b, 1992; Zicovich-Wilson 1994; Fowler 1984; Aquino 1995; Dineykhan 1999; Singh 1984; Montgomery 2002; Laughlin 2002) have been performed for studying the energy levels of compressed atoms. In the current communication we have performed a systematic analysis of the effect of a plasma screening and that of a finite confining radius on the dipole polarizabilities and $2 p, 3 p$ and $4 p$ energy levels for the hydrogen atom, their oscillator strengths and transition probabilities with a view to estimate the order of magnitude of the spectral line shifts under such confinements. Hydrogen atom is chosen as a prototype as it is free from correlation effects and admits of accurate results in a first hand analysis and calculations have already been performed for the hydrogen donor states in spherical quantum dots (Zhu 1990). While studies on the energy levels and other properties of hydrogen using a cutoff radius have been performed by a number of authors (Sommerfeld 1938; Ley-Koo 1979; ZicovichWilson 1994; Fowler 1984; Aquino 1995; Varshni 1997, 1998; Dineykhan 1999), only a single limited calculation by Singh \& Varshni (1984) was performed for the bound states of static screened Coulomb and cut-off Coulomb potentials. Very recently the dynamic dipole polarizability of compressed hydrogen was calculated by Montgomery (2002) for different radii of compression and $1 \mathrm{~s} \rightarrow 2 \mathrm{p}$ transition energy was determined. The recent calculation of Laughlin et al. (2002) on compressed hydrogen atom using a variety of analytical and algebraic methods yields accurate estimate of the ground and excited state energies and wave functions. A brief description of the current method is described in Sect. 2 followed by a discussion of results in Sect. 3.

\section{Method}

The hydrogen atom subjected to a weakly coupled plasma which admits of a Debye type of screening (Akhiezer 1975) in the nuclear potential. In addition the charge cloud is assumed confined in spherical box of radius $R$ which produces an altered boundary condition such that wave function vanishes at the boundary. We assume that the potential energy function (atomic unit is used)

$$
\begin{aligned}
V(r) & =-\frac{Z \mathrm{e}^{-\mu r}}{r} & & \text { for } r \leq R \\
& =\infty & & \text { for } r>R
\end{aligned}
$$

where $\mu$ is a screening parameter due to the surrounding plasma and the quantity $D=\frac{1}{\mu}$ is usually called the Debye radius for a weakly coupled plasma. To get the ground state energy under such a potential one has to solve the Schrodinger eqn.

$\left[-\frac{1}{2} \nabla^{2}+V(r)\right] \psi(\boldsymbol{r})=E_{0} \psi(\boldsymbol{r})$

subject to proper normalization condition. To incorporate the finite boundary condition we assume the radial part of the wave function $\psi(\boldsymbol{r})$ to be of the form

$\psi(r)=(R-r) \chi(r)$.

Here $\chi(r)$ is a radial basis set obtained from a linear combination of Slater type orbitals (STO).

$\chi(r)=\sum_{i} C_{i} r^{n_{i}} \mathrm{e}^{-\rho_{i} r}$

where the exponents $n_{i}, \rho_{i}$ are preassigned and $C_{i}$ are linear variational parameters to be determined from solution of the eigenvalue equation. A structurally simpler but similar basis set was applied by Marin \& Cruz (1992) and also by Varshni (1998). The ground state energy and wave function of the confined hydrogen atom is determined from the iterative solution of the generalized eigenvalue equation

$\underline{\underline{H_{0}}} \underline{\underline{C}}=E_{0} \underline{\underline{S}} \underline{C}$

where $H_{0}$ is the matrix element of the Hamiltonian with respect to the basis set used and $\underline{S}$ is the overlap matrix. The solutions have been obtained with $\overline{\bar{t}}$ respect to different sets of screening parameters $\mu$ and different values of the truncation radii $R$. All the integrals have been evaluated within a domain consistent with the boundary condition. To get excitation properties, a time dependent harmonic perturbation is applied on the system.

$H^{\prime}(\boldsymbol{r}, t)=g(\boldsymbol{r}) \mathrm{e}^{-i \omega t}+g^{\dagger}(\boldsymbol{r}) \mathrm{e}^{+i \omega t}$

where $g(\boldsymbol{r})$ is an one particle perturbation. The angular part of $g(\boldsymbol{r})$ may be chosen to simulate a specific type of excitation. Currently we use a dipolar form of the perturbation. The external perturbation changes the ground state wave function and the correction to first order is given by

$\delta \psi(\boldsymbol{r}, t)=\delta \psi^{-}(\boldsymbol{r}) \mathrm{e}^{-i \omega t}+\delta \psi^{+}(\boldsymbol{r}) \mathrm{e}^{+i \omega t}$.

The radial part of the perturbed admixtures $\delta \psi^{ \pm}(\boldsymbol{r})$ is expanded in terms of STO basis sets similar to that given by Eq. (3)

$\delta \psi^{ \pm}(r)=\sum_{i} d_{i}^{ \pm}(R-r) \eta_{i}(r)$

where $\eta_{i}(r)$ is given by

$\eta_{i}=r_{i}^{n^{\prime}} \mathrm{e}^{-\rho_{i}^{\prime} r}$

with preassigned sets $n_{i}^{\prime}$ and $\rho^{\prime}{ }_{i}$. The linear variational parameters $d_{i}{ }^{ \pm}$are determined from a variational functional (Lowdin 1972)

$J(\phi)=\frac{1}{T} \int_{0}^{T} \frac{\left\langle\phi\left|H-i \frac{\partial}{\partial t}\right| \phi\right\rangle \mathrm{d} t}{\langle\phi \mid \phi\rangle \mathrm{d} t}$ 
subject to optimization condition

$\delta J(\phi)=0$

with respect to variational parameters. Here $|\phi\rangle$ is the total wave function in presence of perturbation and is given by

$\phi(\boldsymbol{r}, t)=N\left[\psi(r)+\delta \psi^{-} \mathrm{e}^{-i \omega t}+\delta \psi^{+} \mathrm{e}^{+i \omega t}\right] \mathrm{e}^{-i E_{0} t}$

$N$ being a normalisation constant and $H$ is the total Hamiltonian

$H=H_{0}+H^{\prime}$.

For hydrogen like systems the functional $J(\phi)$ is given by (up to second order)

$$
\begin{aligned}
J(\phi)= & I\left(\delta 1 s^{-}, \delta 1 s^{-}\right)+\left(E_{0}+\omega\right) S\left(\delta 1 s^{-}, \delta 1 s^{-}\right) \\
& +2\left\langle 1 s|g(r)| \delta 1 s^{-}\right\rangle
\end{aligned}
$$

+ similar terms involving $\delta 1 \mathrm{~s}^{+}$and $-\omega$ in place of $\omega$

where the quantities have their usual significance (Mukherjee 1969). The optimization condition (11) yields sets of decoupled linear equations in $d^{ \pm}$which can be solved by standard techniques. The frequency dependent polarizability value $\alpha(\omega)$ is obtained from the ratio of the expectation value of the time dependent dipole moment to the oscillatory electric field. The static polarizability is given by $\alpha(\omega)_{\omega \rightarrow 0}$. The polarizability $\alpha(\omega)$ passes through poles for certain values of $\omega$ corresponding to the transition energies to various dipole allowed excited states of the system. For a given value of truncation radius $R$, the first three pole positions have been sought for a range of screening parameters $\mu$ from zero to a value for which the energy becomes positive. The procedure is repeated for a number of values of $R$, the range being from $R=\infty$ to a value $R_{0}$ for which the atom becomes unbound given by positive energy solutions. The oscillator strengths and transition probabilities are obtained from standard expressions for the cases for bound transitions (Bethe 1954). The next section deals with the discussion of results.

\section{Result and discussion}

We have chosen confined hydrogen atom to make a detailed analysis on the ground and excited state energy levels, polarizabilities, oscillator strength and transition probabilities. The confinement effect due to (i) Debye screening (ii) finite boundary conditions by using a cut off potential, is analyzed. The energy levels and other properties are calculated systematically first by choosing $R=\infty$ and $\mu=0$ which correspond to free atom case. Then we decreased $R$ step by step to get the effect of reduced cut off parameter and for each $R$, we have chosen different sets of screening parameter $\mu$, always starting with $\mu=0$. In each case the ground state energy under confined condition has been evaluated with a two parameter Slater type representation, the coefficients are obtained from diagonalisation of the Hamiltonian matrix in an iterative manner described earlier. For confined hydrogen atom a two parameter representation is expected to be sufficient for energy convergence. The polarizabilities and transition properties in

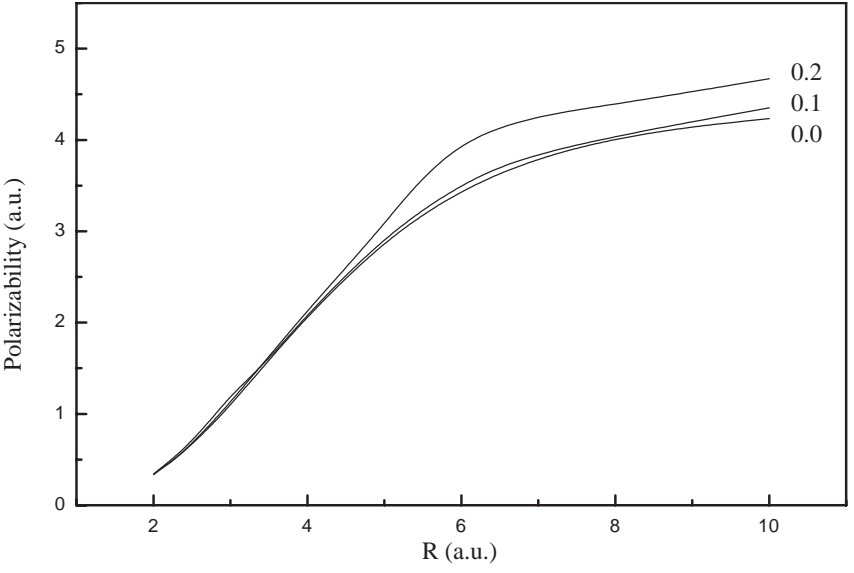

(a)

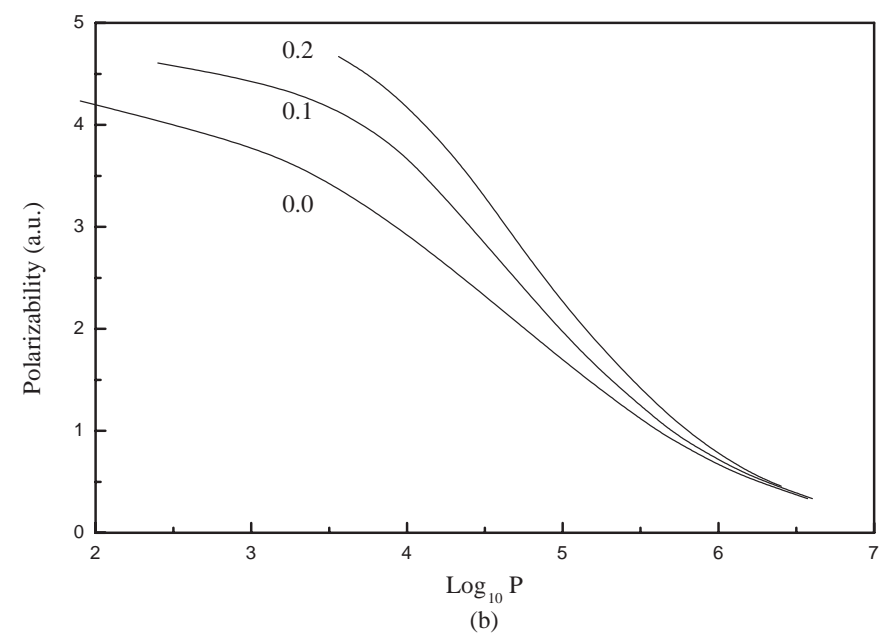

Fig. 1. Plot of polarizability $\left(\alpha_{\mathrm{d}}\right)$ against a) confinement radius $(R)$, b) $\log _{10} \mathrm{P}$ for different Debye screening $(\mu)$.

each case are obtained from a time dependent perturbative calculation. An eight parameter representation of the perturbed wave function is consistently chosen to estimate the transition properties. The length of the basis set and exponents are so chosen as to reproduce the static limit of dynamic polarizability, ground state energy, transition energies to different dipole allowed excited states, oscillator strengths and the transition probabilities for the free hydrogen atom. Results are listed in Table 1. For $R=\infty$ and $\mu=0$ corresponding to the free atom case, our table shows $\alpha=4.4997$ (a.u.) corresponding to the exact value 4.5 a.u. (Miller 1977), $E_{\mathrm{gr}}=-0.5, \triangle E(2 \mathrm{p})=0.375$, $\triangle E(3 \mathrm{p})=0.44444, \triangle E(4 \mathrm{p})=0.46875$ a.u. respectively which are exact up to the places shown (Moore 1949). The oscillator strengths and transition probabilities for $2 p, 3 p$ and $4 p$ excitations are also the same as listed by Wiese et al. (1966). Several features of the different transition properties can be noted from a close look at the Table 1 . The dipole polarizability value increases continuously with increase of screening parameter $\mu$ for a given value of the truncation radius $R$, whereas for a given $\mu$, the polarizability value decreases with decrease of $R$ which simulates stronger confinement. Figure 1a shows plots 
Table 1. Dipole polarizability, Ground state energy $\left(-E_{0}\right)$, Transition energy, Oscillator strength and Transition probability for compressed hydrogen atom under Debye screening $(\mu)$.

\begin{tabular}{|c|c|c|c|c|c|c|c|}
\hline $\begin{array}{c}R \\
\text { (a.u.) }\end{array}$ & $\begin{array}{c}\mu \\
\text { (a.u.) }\end{array}$ & $\begin{array}{c}\text { Dipole } \\
\text { polarizability (a.u.) }\end{array}$ & $\begin{array}{l}-E_{0} \\
\text { (a.u.) }\end{array}$ & Transition & $\begin{array}{c}\text { Transition } \\
\text { energy (a.u.) }\end{array}$ & $\begin{array}{l}\text { Oscillator } \\
\text { strength }\end{array}$ & $\begin{array}{c}\text { Transition } \\
\text { probability }\left(\mathrm{s}^{-1}\right)\end{array}$ \\
\hline \multirow[t]{21}{*}{$\infty$} & 0.00 & 4.4997 & 0.50000 & $1 s \rightarrow 2 p$ & 0.37500 & 0.4160 & $1.87(+9)$ \\
\hline & & $4.5^{a}$ & $0.5^{b}$ & & $0.37500^{b}$ & $0.4162^{c}$ & $1.88(+9)^{c}$ \\
\hline & & & & $\rightarrow 3 \mathrm{p}$ & 0.44444 & 0.0790 & $0.50(+9)$ \\
\hline & & & & & $0.44444^{b}$ & $0.0791^{c}$ & $0.50(+9)^{c}$ \\
\hline & & & & $\rightarrow 4 \mathrm{p}$ & 0.46875 & 0.0290 & $0.20(+9)$ \\
\hline & & & & & $0.46875^{b}$ & $0.0290^{c}$ & $0.20(+9)^{c}$ \\
\hline & 0.01 & 4.5014 & 0.49008 & $\rightarrow 2 \mathrm{p}$ & 0.37482 & 0.4150 & $1.86(+9)$ \\
\hline & & & & $\rightarrow 3 \mathrm{p}$ & 0.44392 & 0.0780 & $0.49(+9)$ \\
\hline & & & & $\rightarrow 4 \mathrm{p}$ & 0.46776 & 0.0280 & $0.20(+9)$ \\
\hline & 0.05 & 4.5412 & 0.45182 & $\rightarrow 2 \mathrm{p}$ & 0.37107 & 0.4000 & $1.76(+9)$ \\
\hline & & & & $\rightarrow 3 p$ & 0.43325 & 0.0660 & $0.39(+9)$ \\
\hline & & & & $\rightarrow 4 \mathrm{p}$ & 0.44922 & 0.0150 & $0.94(+8)$ \\
\hline & 0.08 & 4.6026 & 0.42457 & $\rightarrow 2 \mathrm{p}$ & 0.36545 & 0.3766 & $1.61(+9)$ \\
\hline & & & & $\rightarrow 3 \mathrm{p}$ & 0.41823 & 0.0470 & $0.26(+9)$ \\
\hline & & & & $\rightarrow 4 \mathrm{p}$ & 0.42581 & 0.0062 & $0.38(+8)$ \\
\hline & 0.10 & 4.6577 & 0.40705 & $\rightarrow 2 \mathrm{p}$ & 0.36051 & 0.3561 & $1.48(+9)$ \\
\hline & & & & $\rightarrow 3 \mathrm{p}$ & 0.40546 & 0.0298 & $0.16(+9)$ \\
\hline & 0.15 & 4.8423 & 0.36544 & $\rightarrow 2 \mathrm{p}$ & 0.34433 & 0.2854 & $1.08(+9)$ \\
\hline & 0.20 & 5.0936 & 0.32674 & $\rightarrow 2 \mathrm{p}$ & 0.32263 & 0.1773 & $0.59(+9)$ \\
\hline & 0.25 & 5.41 & 0.29076 & $\rightarrow 2 \mathrm{p}$ & 0.29479 & 0.0383 & $0.11(+9)$ \\
\hline & 1.00 & 8.23 & -0.16598 & $\rightarrow 2 \mathrm{p}$ & 0.01907 & 0.0034 & $0.397(+5)$ \\
\hline \multirow[t]{10}{*}{20} & 0.00 & 4.4628 & 0.50000 & $1 \mathrm{~s} \rightarrow 2 \mathrm{p}$ & 0.37500 & 0.4091 & $1.84(+9)$ \\
\hline & & & & $\rightarrow 3 \mathrm{p}$ & 0.44838 & 0.1068 & $0.69(+9)$ \\
\hline & 0.05 & 4.5005 & 0.45181 & $\rightarrow 2 \mathrm{p}$ & 0.37107 & 0.3925 & $1.73(+9)$ \\
\hline & & & & $\rightarrow 3 \mathrm{p}$ & 0.43908 & 0.1059 & $0.65(+9)$ \\
\hline & 0.1 & 4.6059 & 0.40703 & $\rightarrow 2 \mathrm{p}$ & 0.36053 & 0.3487 & $1.45(+9)$ \\
\hline & & & & $\rightarrow 3 \mathrm{p}$ & 0.41746 & 0.1095 & $0.61(+9)$ \\
\hline & 0.15 & 4.7724 & 0.36540 & $\rightarrow 2 \mathrm{p}$ & 0.34448 & 0.2825 & $1.07(+9)$ \\
\hline & 0.20 & 4.9975 & 0.32666 & $\rightarrow 2 \mathrm{p}$ & 0.32360 & 0.2025 & $0.68(+9)$ \\
\hline & 0.25 & 5.2815 & 0.29063 & $\rightarrow 2 \mathrm{p}$ & 0.29890 & 0.1287 & $0.37(+9)$ \\
\hline & 1.00 & 49.3300 & -0.0213 & & & & \\
\hline \multirow[t]{7}{*}{10} & 0.00 & 4.2343 & 0.499810 & $\rightarrow 2 \mathrm{p}$ & 0.38094 & 0.4382 & $2.03(+9)$ \\
\hline & & $3.977^{f}$ & $0.499999^{d}$ & & $0.38114^{e}$ & & \\
\hline & 0.05 & 4.2652 & 0.45160 & $\rightarrow 2 \mathrm{p}$ & 0.37769 & 0.4299 & $1.96(+9)$ \\
\hline & 0.10 & 4.3516 & 0.40676 & $\rightarrow 2 \mathrm{p}$ & 0.36906 & 0.4091 & $1.78(+9)$ \\
\hline & 0.15 & 4.4875 & 0.36303 & $\rightarrow 2 \mathrm{p}$ & 0.35636 & 0.3890 & $1.55(+9)$ \\
\hline & 0.20 & 4.6702 & 0.32627 & $\rightarrow 2 \mathrm{p}$ & 0.34062 & 0.3488 & $1.29(+9)$ \\
\hline & 1.00 & 18.9700 & -0.029178 & & & & \\
\hline
\end{tabular}

of $\alpha$ against $R$ for three different values of $\mu$. With decrease of $R$, the system becomes more compressed and hence the polarizability decreases. For a given $R$, with increase of $\mu$, both the kinetic energy and potential energy diminish resulting in an increase of $\alpha$ value. When $\mu$ increases for a given $R$ or when $R$ diminishes for a given $\mu$, the total energy monotonically tends towards positive value from bound negative value indicating instability in the system. The cause is entirely different. For example for the free atom case $(R=\infty)$ when $\mu$ increases from zero value, the kinetic energy as well as the absolute value of potential energy diminishes but the potential energy diminishes faster than the kinetic energy resulting in eventual zero energy configuration. As $\mu$ increases further the total energy becomes positive and the system becomes unbound. The polarizability looses its precise meaning. On the other hand for a given $\mu$ (say $\mu=0$ ), with decrease of confinement radius $R$, the atom gets more compressed and the absolute value of the kinetic and potential energy increases but the increase of kinetic energy 
Table 1. continued.

\begin{tabular}{|c|c|c|c|c|c|c|c|}
\hline $\begin{array}{c}R \\
\text { (a.u.) }\end{array}$ & $\begin{array}{c}\mu \\
\text { (a.u.) }\end{array}$ & $\begin{array}{l}\text { Dipole } \\
\text { polarizability (a.u.) }\end{array}$ & $\begin{array}{l}-E_{0} \\
\text { (a.u.) }\end{array}$ & Transition & $\begin{array}{c}\text { Transition } \\
\text { energy (a.u.) }\end{array}$ & $\begin{array}{l}\text { Oscillator } \\
\text { strength }\end{array}$ & $\begin{array}{c}\text { Transition } \\
\text { probability }\left(\mathrm{s}^{-1}\right)\end{array}$ \\
\hline \multirow[t]{5}{*}{7} & 0.00 & 3.8079 & 0.49871 & $\rightarrow 2 \mathrm{p}$ & 0.41122 & 0.5438 & $2.94(+9)$ \\
\hline & 0.05 & 3.8304 & 0.45046 & $\rightarrow 2 \mathrm{p}$ & 0.40889 & 0.5399 & $2.88(+9)$ \\
\hline & 0.10 & 3.8935 & 0.40551 & $\rightarrow 2 \mathrm{p}$ & 0.40261 & 0.5300 & $2.75(+9)$ \\
\hline & 0.15 & 3.9923 & 0.36361 & $\rightarrow 2 \mathrm{p}$ & 0.39329 & 0.5158 & $2.55(+9)$ \\
\hline & 1.00 & 11.26 & -0.04309 & & & & \\
\hline \multirow[t]{5}{*}{6} & 0.00 & 3.4652 & 0.49707 & $\rightarrow 2 \mathrm{p}$ & 0.44151 & 0.6063 & $3.78(+9)$ \\
\hline & 0.05 & 3.4825 & 0.44878 & $\rightarrow 2 \mathrm{p}$ & 0.43957 & 0.6036 & $3.73(+9)$ \\
\hline & 0.10 & 3.5312 & 0.40375 & $\rightarrow 2 \mathrm{p}$ & 0.43433 & 0.5966 & $3.60(+9)$ \\
\hline & 0.15 & 3.6074 & 0.36170 & $\rightarrow 2 \mathrm{p}$ & 0.42648 & 0.5865 & $3.41(+9)$ \\
\hline & 1.00 & 8.4200 & -0.04907 & & & & \\
\hline \multirow[t]{3}{*}{5.50} & 0.00 & 3.2176 & 0.49536 & $1 s \rightarrow 2 p$ & 0.46581 & 0.6438 & $4.46(+9)$ \\
\hline & 0.05 & 3.2319 & 0.44706 & $\rightarrow 2 \mathrm{p}$ & 0.46409 & 0.6416 & $4.42(+9)$ \\
\hline & 1.00 & 7.0000 & -0.05586 & & & & \\
\hline \multirow[t]{3}{*}{5.00} & 0.00 & 2.9051 & 0.49240 & $\rightarrow 2 \mathrm{p}$ & 0.49999 & 0.6855 & $5.48(+9)$ \\
\hline & & & & & $0.50401^{e}$ & & \\
\hline & 0.80 & 4.7600 & -0.00304 & & & & \\
\hline \multirow[t]{3}{*}{2.00} & 0.00 & 0.3344 & 0.12210 & $\rightarrow 2 \mathrm{p}$ & 1.69812 & 0.9643 & $8.88(+9)$ \\
\hline & & $0.338^{f}$ & $0.125^{g}$ & & $1.70102^{e}$ & & \\
\hline & 0.20 & 3.3600 & -0.06197 & & & & \\
\hline \multirow[t]{2}{*}{1.00} & 0.00 & 0.0291 & -2.38187 & $\rightarrow 2 \mathrm{p}$ & 5.84138 & 0.9920 & $1.08(+12)$ \\
\hline & & & & & $5.85915^{e}$ & & \\
\hline
\end{tabular}

${ }^{a}$ Ref. Miller; ${ }^{b}$ Ref. Moore; ${ }^{c}$ Ref. Wiese; ${ }^{d}$ Ref. Laughlin; ${ }^{e}$ Ref. Montgomery; ${ }^{f}$ Ref. Aquino; ${ }^{g}$ Ref. Ley-Koo. Note: the data for comparison have been truncated in appropriate cases.

is more than the increase of potential energy. Eventually at a given value of $R$, the total energy becomes zero resulting in pressure ionization. Further decrease of $R$ results in unbound states, for which polarizability again looses its meaning. With decrease of truncation radius $R$ the charge cloud gets pressurized. We have calculated the pressure on the atom due to truncation of the wave function at finite radius $R$ by using the relation (Hirschfelder 1954)

$P=\frac{1}{4 \pi R^{3}}\left[2 E_{0}-\langle V\rangle\right]$.

The value of $P$ has been calculated from the estimated values of $E_{0}$ and $\langle V\rangle$. In Fig. $1 \mathrm{~b}$ a plot of the polarizability values against logarithm of pressure is given for different values of screening parameters $\mu$. The features show that for a given pressure, the polarizability value $\alpha$ is greater for larger screening parameters $\mu$. The curves, however tend to converge towards the ionization pressure for which $E_{0}=0$, for each Debye parameter. Our estimate shows that the ionization pressure corresponding to $\mu=0,0.1$ and 0.2 (a.u.) are respectively $6.09 \times 10^{6}, 4.49 \times 10^{6}$ and $3.20 \times 10^{6}$ in atmos corresponding to the confinement radii as $1.8373,1.9604$ and 2.1068 a.u. The ionization pressure calculated by us in conformity with the estimate due to Ley-Koo \& Rubinstein (1979) with whom we have checked our calculated pressure for $R=2$ a.u. In Fig. 2 we plotted the change

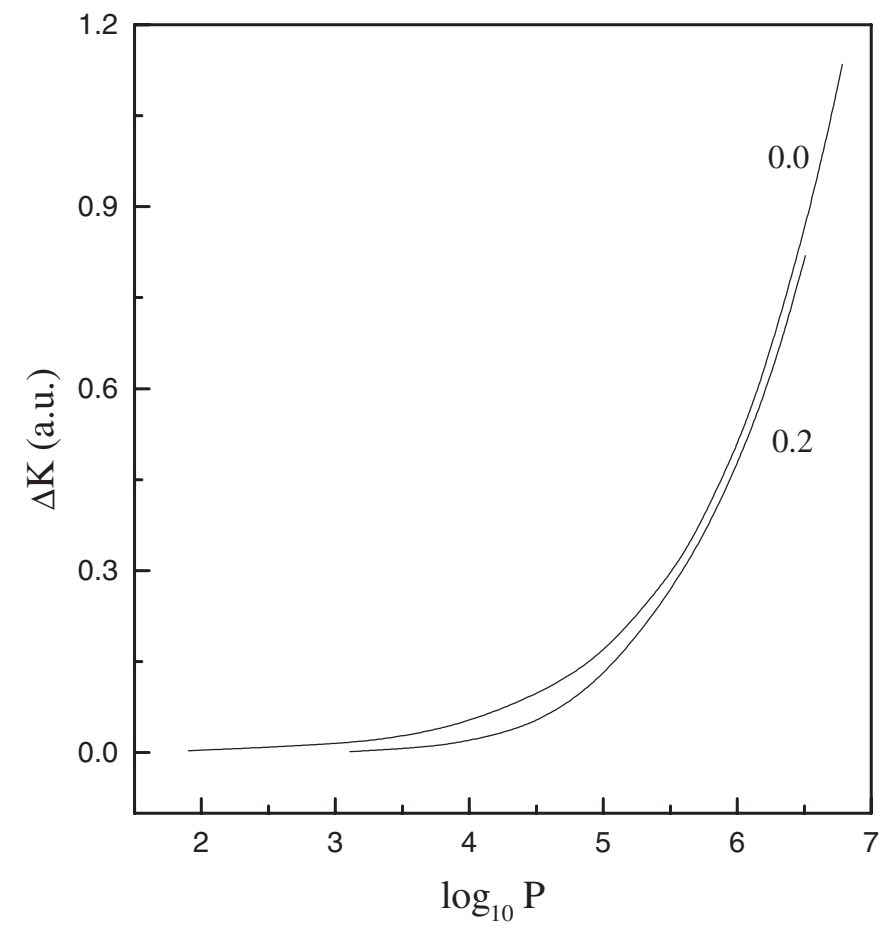

Fig. 2. Variation of change in kinetic energy ( $\triangle K$ in a.u.) with logarithm of pressure at different screening. 


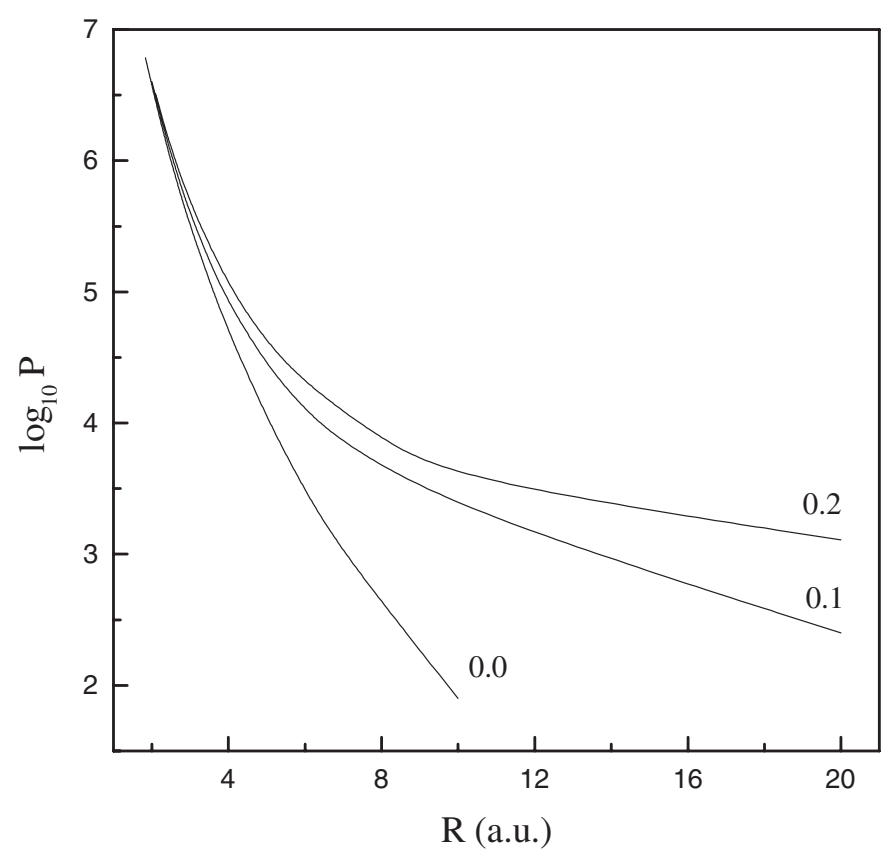

Fig. 3. Plot of $\log _{10} P$ against confinement radius $R$ at different screening.

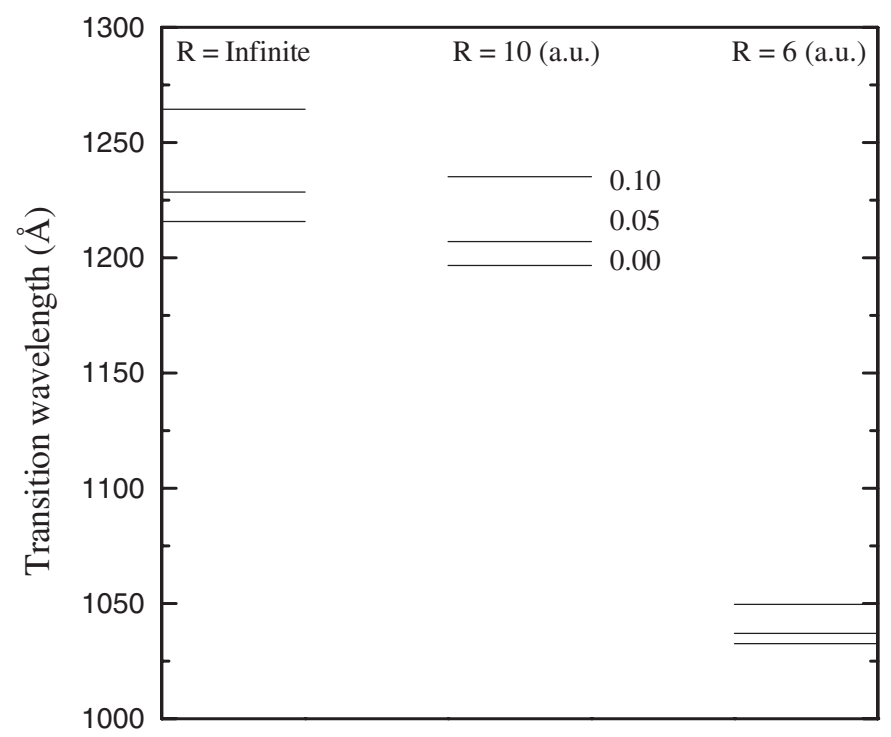

Fig. 4. Transition wave length for $2 p$ state of compressed hydrogen atom under different confinement radii and Debye shielding $(\mu)$.

in kinetic energy $\triangle K=K(R)-K(R=\infty)$ with respect to logarithm of pressure for two different screening parameters. The behavior of kinetic energy with respect to $R$ is similar for different Debye screening. The behavior for $\mu=0$ is similar to that plotted by Ludena $(1977,1978)$ for helium like ions. To have a feeling for the pressure generated due to the confinement, a plot of $\log _{10} P$ against $R$ is shown in Fig. 3 for three different values of $\mu$. It is noted that the effect of Debye shielding on the calculated pressure is less sensitive at lower values of $R$ while that is much more prominent for higher values of $R$.

For a given $\mu$, the transition energy to different excited states increases with decrease of $R$, i.e., with more and more

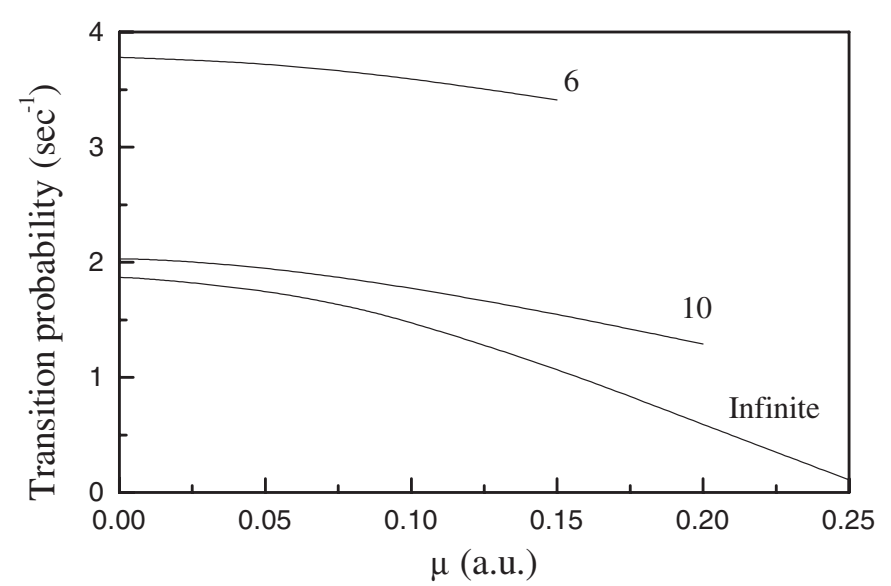

(a)

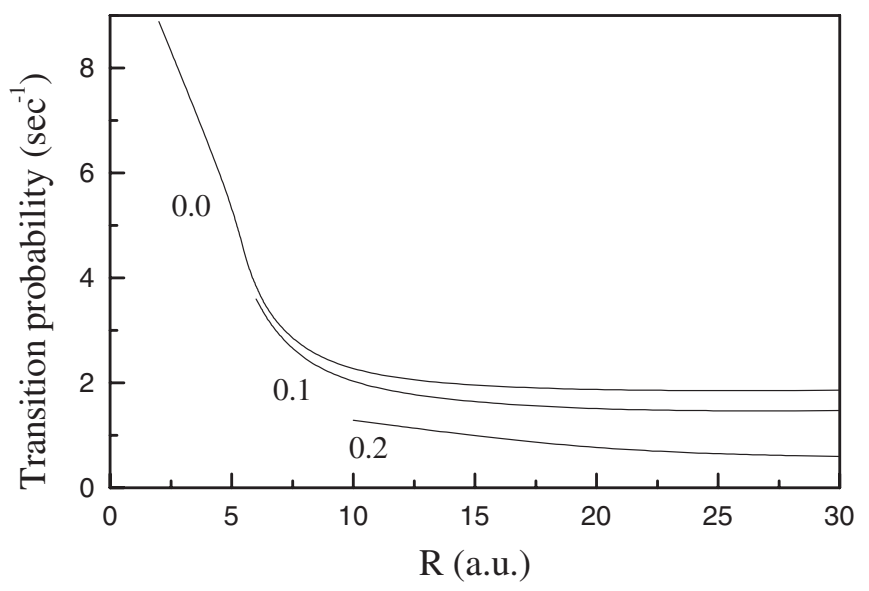

(b)

Fig. 5. Variation of transition probability (scaled) with a) $\mu$ at different radii $(R)$ of the spherical box, b) the radius of the spherical box at different $\mu$.

compression. However, for given $R$, the transition energy diminishes with increase of $\mu$ value. The wavelength in $\AA$ for the 2 p transition is schematically shown in Fig. 4 for different $R$ and $\mu$. The general trend in the change of wave length is clearly observed. A typical example for $R=10$ a.u. and $\mu=0.1$ a.u. the shift $\Delta \lambda \sim 1.96 \mathrm{~nm}$. Experiments performed for different atoms in liquid helium show shifts of this order (Tabbert 1995). The oscillator strength and transition probability for dipole allowed transitions have been evaluated using standard formula (Bethe 1954). The oscillator strength and transition probability values diminish along increasing $\mu$ for a given truncation radius $R$, while they gradually increase with decrease of $R$ values for a given $\mu$. Figure 5 a shows the plot of transition probability for the excitation $1 \mathrm{~s} \rightarrow 2 \mathrm{p}$ against $\mu$ for three different confinement radii $R$, while in Fig. 5b, the same is plotted against $R$ for three different values of screening parameters $\mu$. Different trend is observed for the two cases. As the energy becomes less negative either when $\mu$ is increased for a given $R$ or when $R$ is decreased for a given $\mu$ the ionization potential gets lowered and the number of excited states becomes finite. To have an idea about the behavior of the excited state wave functions 


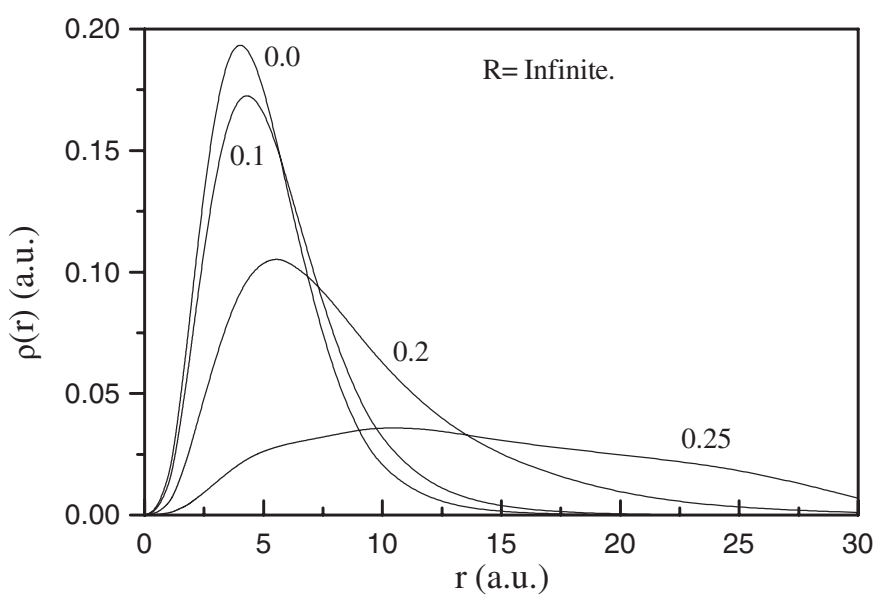

(a)

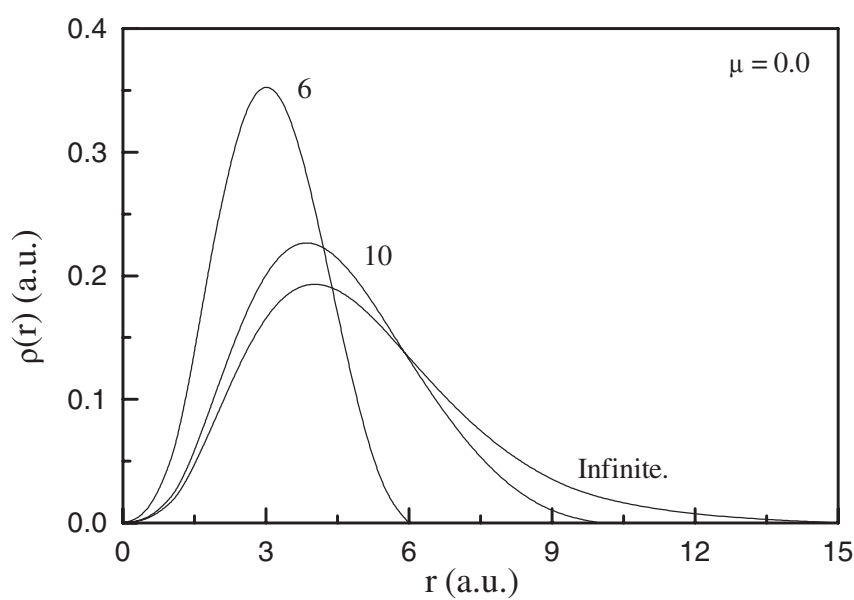

(b)

Fig. 6. Plot of charge density for $2 p$ state a) under different Debye screening ( $\mu$ in a.u.) with no pressure, i.e., $R=$ Infinite and $\mathbf{b}$ ) under different compression with no Debye shielding, i.e., $\mu=0$.

under confinement, we plotted in Fig. 6a the charge density for the $2 \mathrm{p}$ wave function calculated by our method against $r$ for sets of Debye parameters for $R=\infty$ while in Fig. $6 \mathrm{~b}$ the plots for different confinement radii for the Debye parameter $\mu=0$ are given. An overall flattening of the charge density is noted for increase of $\mu$ value for $R=\infty$ while the charge density is squeezed for decrease of truncation radius $R$ for $\mu=0$. Similar plots are given in Figs. 7, 8a and 8b for finite $R$ and $\mu$ values. The figures show interesting feature of the charge densities. The trend is similar for charge densities of the excited $3 p$ and $4 p$ states.

Acknowledgements. PKM would like to thank the Max-Planck Institute for Astrophysics, Garching, for financial support of his research visit to the institute where part of the work was performed. He would also like to thank the Council of Scientific and Industrial Research (CSIR), Govt. of India for the research grant No. (03)/(0888)/99/EMR II.

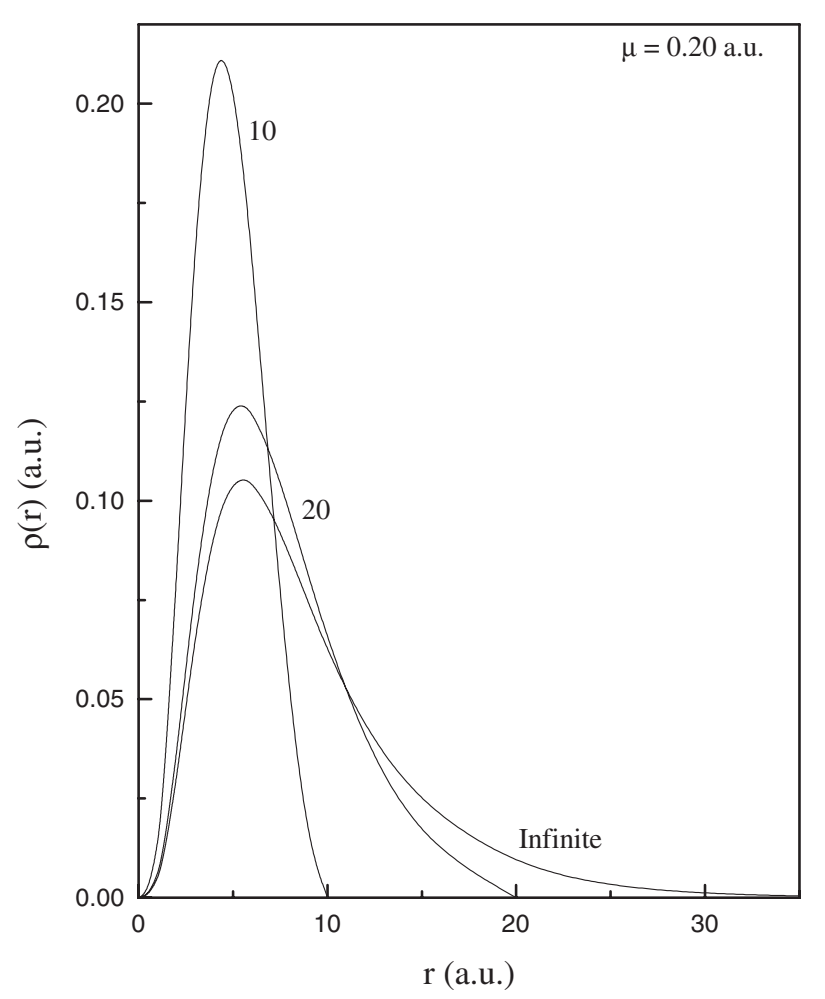

Fig. 7. Plot of charge density at different confinement with $\mu=0.2$ a.u.

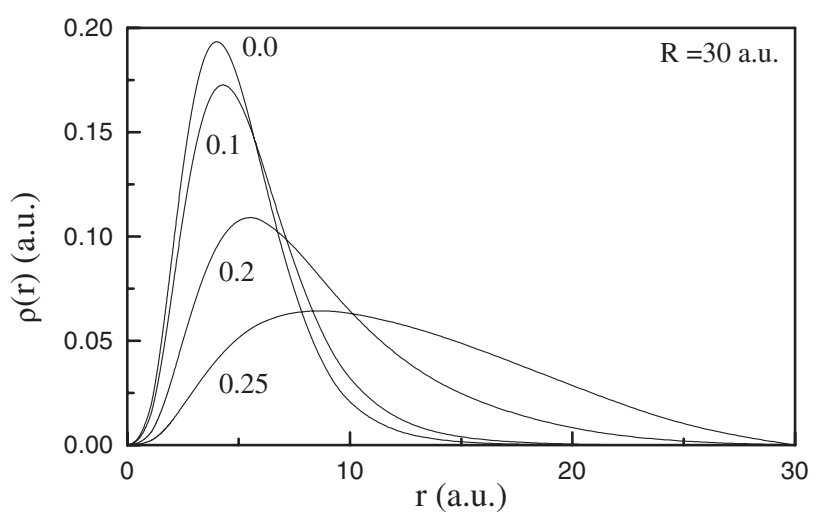

(a)

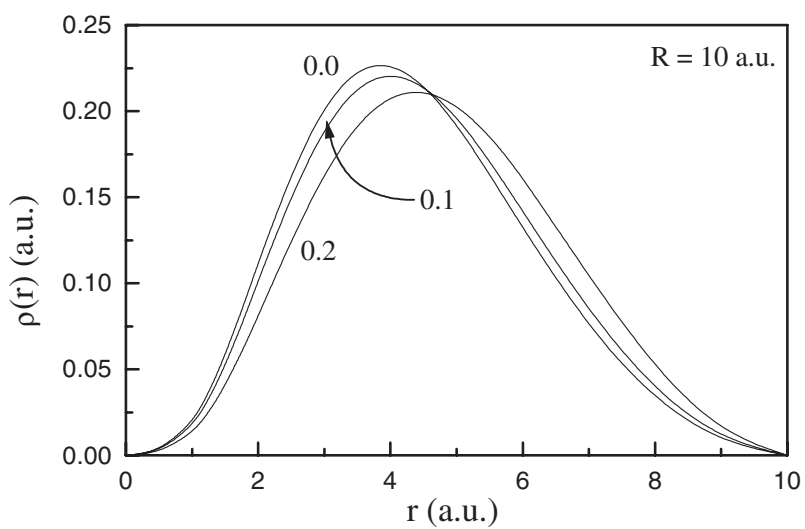

(b)

Fig. 8. Plot of charge density at different screening with confinement radii a) $R=30$ and b) $R=10$ a.u. respectively. 


\section{References}

Akhiezer, A. I., Akhiezer, I. A., Polovin, R. V., Sitenko, A. G., \& Stepanov, K. N. 1975, Plasma Electrodynamics, vol. 1, Linear Theory (Pergamon), 1

Aquino, N. A. 1995, Int. J. Quantum Chem., 54, 107

Arndt, M., Kanorski, S. I., Weis, A., \& Hänsch, T. W. 1993, Phys. Lett. A, 174, 298

Bauer, H., Beau, M., Fischer, J., et al. 1990, Physica B, 165 \& 166, 137

Bauer, H., Beau, M., Friede, B., et al. 1990, Phys. Lett. A, 146, 134

Beau, M., Günther, H., Putlitz, G. zu, \& Tabbert, B. 1996, Z. Phys. B, 101,253

Beijersbergen, J. H. M., Hui, Q., \& Takami, M. 1993, Phys. Lett. A, 181,393

Bethe, H. A., \& Salpeter, E. E. 1954, Quantum Mechanics of One and Two Electron Atoms (Springer, Berlin)

Connerade, J. P., Dolmatov, V. K., \& Lakshmi, P. A. 2000, J. Phys. B, At. Mol. Opt. Phys., 33, 251 and references there in

David Singh, \& Varshni, Y. P. 1984, Phys. Rev. A, 29, 2895

Dineykhan, M., \& Nazmitdinov, R. G. 1999, Physics of Atomic Nuclei, 62, 138

Fowler, P. W. 1984, Mol. Phys., 53, 865

Gordon, E. B., Mezhop-Deglin, L. P., \& Pugachev, O. F. 1974, JETP Lett., 19, 63

Gordon, E. B., Pelmenev, A. A., Pugachev, O. F., \& Khmelenko, V. V. 1983, JETP Lett., 37, 283

Gordon, E. B., Khmelenko, V. V., Pelmenev, A. A., et al. 1993, Chem. Phys., 170, 411

Günther, H., Beau, M., Foerste, M., et al. 1995, Z. Phys. B, 98, 395

Gupta, U., \& Rajagopal, A. K. 1982, Phys. Rep., 87, 259

Hickman, A. P., Steets, W., \& Lane, N. F. 1975, Phys. Rev. B, 12, 3705

Hiroike, K., Kestner, N. R., Rice, S. A., \& Jortner, J. 1965, J. Chem. Phys., 43, 2625

Hirschfelder, J. O., Curtis, C. F., \& Bird, R. B. 1954, Molecular Theory of Gases and Liquids (John Wiley), 264

Jaskolski, W. 1996, Phys. Rep., 271, 1 and references there in

Kanorski, S. I., Arndt, M., Dziewior, R., Weis, A., \& Hänsch, T. W. 1994, Phys. Rev. B, 49, 3645

Kinoshita, T., Fukuda, K., Takahashi, Y., \& Yabuzaki, T. 1995, Phys. Rev. A, 52, 2707
Laughlin, C., Burrows, B. L., \& Cohen, M. 2002, J. Phys. B, At. Mol. Opt. Phys., 35, 701

Ley-Koo, E., \& Rubinstein, S. 1979, J. Chem. Phys., 71, 351

Lowdin, P. O., \& Mukherjee, P. K. 1972, Chem. Phys. Lett, 14, 1

Ludena, E. V. 1977, J. Chem. Phys., 66, 468

Ludena, E. V. 1978, J. Chem. Phys., 69, 1770

Marin, J. L., \& Cruz, S. A. 1991a, Am. J. Phys., 59, 931

Marin, J. L., \& Cruz, S. A. 1991b, J. Phys. B, At. Mol. Opt. Phys., 24, 2899

Marin, J. L., \& Cruz, S. A. 1992, J. Phys. B, At. Mol. Opt. Phys., 25, 4365

Michels, A., de Boer, J., \& Bijl, A. 1937, Physica, 4, 981

Miller, T. M., \& Bederson, B. 1977, in Adv. At. Mol. Phys., ed. D. R. Bates, \& B. Bederson (Acad. Press.), vol. 13, 1

Montgomery, H. E. 2002, Chem. Phys. Lett., 352, 529

Moore, C. E. 1949, Natl. Bur. Std. (U.S.) Circ., 467, 1

Mukherjee, P. K., Sengupta, S., \& Mukherji, A. 1969, J. Chem. Phys., 51,1397

Ray, D., \& Mukherjee, P. K. 1998, Eur. Phys. J. D, 2, 89

Ray, D., \& Mukherjee, P. K. 1998, J. Phys. B, At. Mol. Opt. Phys., 31, 3479

Seaton, M. J. 1987, J. Phys. B, 20, 6363

Sommerfeld, A., \& Welker, H. 1938, Ann. Phys., 32, 56

Tabbert, B., Beau, M., Ficscher, J., Putlitz, G. zu, \& Schrech, H. 1994, Phys. B, 194, 731

Tabbert, B., Beau, M., Günther, H., et al. 1995, Z. Phys. B, 97, 425

Tabbert, B., Günther, H., \& Putlitz, G. zu 1997, J. Low. Temp. Phys., 109,653

Varshni, Y. P. 1997, J. Phys. B, At. Mol. Opt. Phys., 30, L589

Varshni, Y. P. 1998, J. Phys. B, At. Mol. Opt. Phys., 31, 2849

Winkler, P. 1996, Phys. Rev. E, 53, 5517

Wiese, W. L., Smith, M. W., \& Glennon, B. M. 1966, in Atom. Transn. Prob. vol. 1, NSRDS, NBS-4, 2

Yabuzaki, T., Fujisaki, A., Sano, K., Kinoshita, T., \& Takahashi, Y. 1992, Atom. Phys., 13, ed. H. Walther, \& T. W. Hänsch (North Holland, N. Y.)

Zhu, J. L., Xiong, J. J., \& Gu, B. L. 1990, Phys. Rev. B, 41, 6001

Zicovich-Wilson, C., Planelles, J. H., \& Jaskolski, W. 1994, Int. J. Quantum. Chem., 50, 429 